\title{
Developmental Differences of Structural Connectivity and Effective Connectivity in Semantic Judgments of Chinese Characters
}

\author{
Li-Ying Fan ${ }^{1,2,3}$, Yu-Chun Lo ${ }^{4,5,6}$, Yung-Chin Hsu ${ }^{4}$, Yu-Jen Chen ${ }^{4}$, Wen-Yih Isaac Tseng $4,7,8 *$ \\ and Tai-Li Chou ${ }^{2,7,9 *}$ \\ 'Department of Education, National Taipei University of Education, Taipei, Taiwan, ${ }^{2}$ Department of Psychology, National \\ Taiwan University, Taipei, Taiwan, ${ }^{3}$ Department of Thanatology and Health Counseling, National Taipei University of Nursing \\ and Health Sciences, Taipei, Taiwan, ${ }^{4}$ Institute of Medical Device and Imaging, National Taiwan University College of \\ Medicine, Taipei, Taiwan, ${ }^{5}$ The Ph.D. Program for Neural Regenerative Medicine, College of Medical Science and \\ Technology, Taipei Medical University, Taipei, Taiwan, ${ }^{6}$ Research Center of Brain and Consciousness, Taipei Medical \\ University, Taipei, Taiwan, 'Graduate Institute of Brain and Mind Sciences, National Taiwan University, Taipei, Taiwan, \\ ${ }^{8}$ Molecular Imaging Center, National Taiwan University, Taipei, Taiwan, ${ }^{9} \mathrm{Center}$ for Advanced Study in the Behavioral \\ Sciences, Stanford University, Stanford, CA, United States
}

\section{OPEN ACCESS}

Edited by:

S. H. Annabel Chen,

Nanyang Technological University,

Singapore

Reviewed by:

Nestor Vinas-Guasch,

Nanyang Technological University,

Singapore

Blake Warren Johnson, Macquarie University, Australia

*Correspondence: Wen-Yih Isaac Tseng wytseng@ntu.edu.tw Tai-LiChou

t/chou25@ntu.edu.tw

Specialty section: This article was submitted to Speech and Language, a section of the journal Frontiers in Human Neuroscience

Received: 20 February 2020 Accepted: 28 May 2020 Published: 30 June 2020

Citation:

Fan L-Y, Lo Y-C, Hsu Y-C, Chen Y-J, Tseng W-YI and Chou T-L (2020) Developmental Differences of Structural Connectivity and Effective Connectivity in Semantic Judgments of Chinese Characters. Front. Hum. Neurosci. 14:233. doi: 10.3389/fnhum.2020.00233
Previous studies have investigated the developmental differences of semantic processing regarding brain activation between adults and children. However, little is known about whether the patterns of structural connectivity and effective connectivity differ between adults and children during semantic processing. Functional magnetic resonance imaging (fMRI), diffusion spectrum imaging (DSI), and dynamic causal modeling (DCM) were used to study the developmental differences of brain activation, structural connectivity, and effective connectivity during semantic judgments. Twenty-six children (8- to 12-yearolds) and 26 adults were asked to indicate if character pairs were related in meaning. Compared to children, adults showed greater activation in the left ventral inferior frontal gyrus (IFG) and left middle temporal gyrus (MTG). Also, adults had significantly greater structural connectivity in the left ventral pathway (inferior frontal occipital fasciculus, IFOF) than children. Moreover, adults showed significantly stronger bottom-up effects from left fusiform gyrus (FG) to ventral IFG than children in the related condition. In conclusion, our findings suggest that age-related increases in brain activation (ventral IFG and MTG), IFOF, and effective connectivity (from FG to ventral IFG) might be associated with the bottom-up influence of orthographic representations on retrieving semantic representations for processing Chinese characters.

Keywords: meaning, semantics, structural connectivity, effective connectivity, development

\section{INTRODUCTION}

Previous functional and structural studies have proposed that a dual-stream model for the language network includes the ventral and dorsal pathways that connect the functionally relevant frontal and temporal regions (Saur et al., 2008). The dorsal pathway connects the opercular part of the IFG (dorsal IFG) and the superior temporal gyrus (STG) via the arcuate fasciculus (AF) and the superior longitudinal fasciculus (SLF), while the ventral pathway connects the orbital part of IFG (ventral IFG), the middle temporal gyrus (MTG), and the fusiform gyrus (FG) 
via the inferior frontal-occipital fasciculus (IFOF) and the inferior longitudinal fasciculus (ILF; Saur et al., 2008). Relevance to the structural division, previous studies have also suggested functional differences for the sub-parts of the left inferior frontal gyrus (IFG). The dorsal region of the left IFG is found to process phonological information, while the ventral region of the left IFG is associated with semantic processing (Poldrack et al., 1999; Liu et al., 2009).

Regarding developmental differences (ranging from 8 to 20 years) in brain activation, two major brain regions have been suggested to be associated with semantic processing as a network. These two brain regions include: the left ventral IFG and left MTG (Lau et al., 2008). The ventral IFG is associated with controlling semantic retrieval (Lau et al., 2008), while the left MTG is considered to be related to the storage of lexical representations (Hickok and Poeppel, 2007; Martin, 2007). Using a semantic association task, similar activations in the ventral IFG and the MTG have been observed in Chinese adults (Dong et al., 2005; Booth et al., 2006; Chou et al., 2009b) and children (Chou et al., 2009a). A child study has shown that children have similar activation in the bilateral inferior frontal gyri and left MTG as reported by previous studies of adults for printed words (Chou et al., 2006).

To investigate developmental differences in structural connectivity, diffusion tensor imaging (DTI) has been used to obtain information about neural fiber orientations. Previous DTI studies have demonstrated age-related fractional anisotropy (FA) changes (ranging from 5.6 to 29.2 years) in the genu and splenium of the corpus callosum, the inferior and SLF, the inferior and superior fronto-occipital fasciculus, cingulum, uncinate fasciculus, and corticospinal tract (Lebel et al., 2008). The age-related increase in the volume of white matter and FA has been interpreted as evidence of reflecting continued axonal myelination during childhood and adolescence (Paus et al., 2008; Schmithorst and Yuan, 2010). Also, a previous developmental study has shown that children have lower GFA in dorsal and ventral pathways than adults (Brauer et al., 2013). However, DTI may provide false orientations for crossing fibers because the diffusion model of DTI assumes Gaussian distribution of water molecular diffusion within each voxel. Due to the limitation of DTI, DTI tractography may not provide appropriate fiber pathways, especially in the frontal regions (Wedeen et al., 2005). To resolve diffusion profiles in different directions, diffusion spectrum imaging (DSI) acquires hundreds of diffusion-weighted images corresponding to evenly-spaced grid points in 3-D q-space (Wedeen et al., 2005). Since DSI is more sensitive to crossing fibers; it is used in the present study to detect the developmental differences in structural connectivity between adults and children.

To investigate the developmental differences in effective connectivity, dynamic causal modeling (DCM) was used. Previous studies have suggested that effective connectivity measures such as DCM (Friston et al., 2003; Stephan et al., 2010) can estimate inter-regional relationships in a neural network, for example, the direction of the causal influence and its change due to experimental interventions. Our previous work on effective connectivity has provided evidence to support that the left IFG is functionally connected with the left MTG during semantic judgments (Fan et al., 2010; Fan and Chou, 2012). Also, previous studies have suggested that the linkage of different modulatory effects between the top-down controlled retrieval and the bottom-up automatic processing interacts to mediate the access of lexical-semantic knowledge in the MTG (Rossell et al., 2001; Cohen et al., 2004). However, to the best of our knowledge, no study has investigated the developmental differences of semantic processing using effective connectivity.

Previous studies have combined data from DTI and functional magnetic resonance imaging (fMRI) to investigate brain injuries and the organization of brain functions. A combination of techniques can provide additional information about brain organization which might provide different conclusions from an approach using single imaging modality (Werring et al., 1998, 1999; Wieshmann et al., 2001; Olesen et al., 2003). To date, no study has investigated developmental differences in brain activation, structural connectivity, and effective connectivity to understand the underlying mechanisms of semantic processing between adults and children in one study. Therefore, in the present study, we used task fMRI to examine the brain activation of semantic processing, DSI to assess the microstructural integrity, and DCM to examine the directional influences among brain regions between two groups. By combining multiple modalities, our goal was to provide a complete insight into the function and structure of the developmental differences between adults and children during semantic processing in Chinese. Besides, we examined the correlation between various pertinent factors and age in months across all participants. We hypothesized that functional activation, microstructural integrity, and effective connectivity in adults might be greater than those in children and that the degree of maturation could be revealed by examining the correlation between age and behavioral performance, structural connectivity, and effective connectivity.

\section{MATERIALS AND METHODS}

\section{Participants}

Twenty-six adults $[$ mean age $=25.9$ years, standard deviation $(\mathrm{SD})=4.8$ years, 14 males ) and 26 children (mean age $=9.8$ years, $\mathrm{SD}=1.5$ years, age range from 8 - to 12 -year-olds, 14 males) participated in the MRI experiment. All participants were given an interview to ensure that they met the following inclusion criteria: (1) native Mandarin-Chinese speakers; (2) righthandedness; (3) free of neurological disease or psychiatric disorders; (4) no history of language or reading disabilities; (5) not taking medication affecting the central nervous system; and (6) no learning difficulty. Participants who had a clinical diagnosis of any other psychiatric disorders were excluded from the present study. After receiving detailed information on the experimental purpose and administration, informed consent was obtained. The informed consent procedures were approved by the Institutional Review Board of National Taiwan University Hospital. Both fMRI and DSI data were collected to examine developmental differences in brain activation, 
effective connectivity, and structural connectivity, respectively. The present study was approved by and following the guidelines of the local ethics committee (Research Ethics Committee at National Taiwan University Hospital).

\section{Functional MRI Acquisition and Analysis}

\section{Stimuli and Procedures}

Character pairs were divided into the semantically related and unrelated conditions (Chou et al., 2009b). Forty-eight character pairs were semantically related according to their free association values (mean $=0.14, \mathrm{SD}=0.13$, ranging from 0.73 to 0.01 ; Hue et al., 2005). Twenty-four character pairs were semantically unrelated with zero association values. Several lexical variables were controlled across related and unrelated conditions. First, all characters were monosyllabic. Second, the first and second characters did not share radicals. Third, the first and second characters together did not form a word ( $\mathrm{Wu}$ and Liu, 1987; Sinica, 1998). Fourth, characters were matched for visual complexity (in terms of strokes per character) across conditions. Fifth, characters were matched for frequency across conditions (Wu and Liu, 1987). Sixth, the number of nouns (48-50\%), verbs (23\%), and adjectives (21-27\%), based on their most frequent usage in Academia Sinica balanced corpus (Sinica, 1998), was matched across conditions. The correlation of character frequency or the measure of semantic relation (Chou et al., 2009a; Lee et al., 2009) with association strength was not significant indicating that association effects should not be due to frequency or semantic relation differences $(r=-0.02$, $p>0.05$ and $r=-0.05, p>0.05$, respectively).

In the meaning judgment task, two visual Chinese characters were presented sequentially (Chou et al., 2009a,b). The participant had to determine whether the character pairs were related in meaning. Trials lasted $4,500 \mathrm{~ms}$ and consisted of a solid square $(500 \mathrm{~ms})$, followed by the first character (800 ms), a $200 \mathrm{~ms}$ blank interval, and the second character (3,000 ms). The participants responded during the presentation of the second character. They were instructed to quickly and accurately make a button press with their right hand for "yes" to related pairs and "no" to unrelated pairs (Chou et al., 2009a,b). The stimuli were presented in an event-related design fashion. In the present study, the Optseq was used for automatically randomizing the sequence order and varying the inter-stimulus-interval (ISI) for rapid-presentation event-related fMRI experiments. The Optseq allowed more stimuli to be included within a given scanning interval at the cost of assuming that the overlap in the hemodynamic responses would be linear (http://surfer.nmr.mgh.harvard.edu/optseq, written by D. Greve, Charlestown, MA). In addition, previous fMRI studies using the OptSeq have got fairly stable findings across studies (Burock et al., 1998; Chou et al., 2006, 2009a,b; Bitan et al., 2009; Fan et al., 2010; Cao et al., 2011; Walter and Dassonville, 2011; Fan and Chou, 2012; Devereux et al., 2013; Mei et al., 2013; Saggar et al., 2014).

There were two kinds of control tasks. The perceptual control had 24 pairs of non-characters. Non-characters were created by replacing radicals of real characters with other radicals that did not form real Chinese characters such as “狌," “硡,” and
“粉” (Wu and Chen, 2000). For the perceptual controls, trials consisted of a solid square $(500 \mathrm{~ms})$, followed by the first non-character $(800 \mathrm{~ms})$, a $200 \mathrm{~ms}$ blank interval, and the second non-character $(3,000 \mathrm{~ms})$. Participants determine whether the pair of stimuli were identical or not by pressing a yes or no button (Chou et al., 2009a,b). In the perceptual task, the non-characters were presented in different font sizes to encourage participants to perform the task based on the recognition of low-level visual similarity (Chou et al., 2009b). The second control task involved 24 baseline events. The participant was instructed to press a button when a solid square $(1,300 \mathrm{~ms})$ at the center of the visual field turned to a hollow square $(3,000 \mathrm{~ms})$ after a blank interval (200 ms; Chou et al., 2009a,b). The total number of trials was 120, and all conditions had the same 4,500 ms duration.

\section{fMRI Data Acquisition}

All participants received two practice sessions (one outside the scanner and the other one in the scanner) to ensure that they understood the task. The stimuli used for the practice sessions and the fMRI task were different. Each participant had to reach at least $80 \%$ accuracy for each practice session before entering the MRI task. In addition to the practice sessions, the children received a two-phase training session outside the scanner. In the first phase, the children and their parents were provided a brief introduction to each step of the scanning procedures. This phase was designed to help them understand the research aims to undergo the MRI investigation and the requirement to minimize head motion. They also received the samples of MRI sounds to experience the MRI task beforehand. In the second phase, the children were given a brief tour to the MRI environment, including looking at the magnet, MRI head coil, scanner bed, video goggles, and response box. After the tour, they entered the MRI scanning session. Earplugs were given to them to reduce noise. They lay in the scanner with a vacuum pillow to restrict head movement. Participants viewed visual stimuli projected onto a screen via a mirror attached to the head coil. Each participant performed two functional runs (Chou et al., 2009a,b). Images were acquired using a 3 Tesla Siemens Tim-Trio scanner with a 32-channel head coil. Each run took 4.7 minutes. Functional images were interleaved in an ascending order and were collected parallel to the anterior commissureposterior commissure plane. The scanning parameters were the following: repetition time $(\mathrm{TR})=2000 \mathrm{~ms}$; echo time $(\mathrm{TE})=$ $24 \mathrm{~ms}$; flip angle $=90^{\circ}$; matrix size $=64 \times 64$; field of view $=$ $25.6 \mathrm{~cm}$ slice thickness $=3 \mathrm{~mm}$; number of slices $=34$. A high-resolution, T1-weighted three dimensional image was also acquired (Magnetization Prepared Rapid Gradient Echo, MP$\mathrm{RAGE} ; \mathrm{TR}=2300 \mathrm{~ms} ; \mathrm{TE}=2.98 \mathrm{~ms}$; flip angle $=9^{\circ}$; matrix size $=$ $256 \times 256$; field of view $=25.6 \mathrm{~cm}$; slice thickness $=1 \mathrm{~mm}$ ). The orientation of the 3D image was identical to the functional slices. The task administration sequence was counterbalanced.

\section{Conventional fMRI Analysis}

Data analysis was performed using SPM8 (Statistical Parametric Mapping) with MATLAB version (Penny et al., 2007). The functional images were corrected for differences in sliceacquisition time to the middle slice of volume and were 
realigned to the first volume in the scanning session using affine transformations. No participant had more than $3 \mathrm{~mm}$ of movement in any plane. Co-registered images were normalized to the MNI (Montreal Neurological Institute) average template. Statistical analyses were calculated on the smoothed data $(8 \mathrm{~mm}$ isotropic Gaussian kernel), with a high pass filter (128 s cut-off period) to remove low-frequency artifacts.

Data from each participant were entered into a general linear model using an event-related analysis procedure (Josephs and Henson, 1999). Character pairs were treated as individual events for analysis and modeled using a canonical Hemodynamic Response Function (HRF), and the stimulus onsets of the four experimental conditions (as a stick function) were convolved with the canonical HRF. There were four event types: related, unrelated, perceptual, and baseline. Thus, there were four regressors for these four experimental conditions in the General Linear Models (GLM). Parameter estimates from contrasts of the canonical HRF in single-subject models were entered into a random-effects analysis using one-sample $t$-tests across all participants to determine whether activation during a contrast was significant (i.e., parameter estimates were reliably greater than 0). Within each group, we used the contrast of the word (related and unrelated conditions) vs. baseline conditions in a whole-brain analysis (Liu et al., 2010). Based on common activation areas of within-group analyses for both groups, we conducted between-group comparisons. Also, for the contrasts between groups, we used the WFU PickAtlas ${ }^{1}$ to define ROI masks by selecting the anatomical masks of the left ventral IFG and left MTG based on our a priori hypothesis. The masks were used to control for multiple comparisons at $p<0.05$ family wise error (FWE) corrected at the voxel level. For both the within-group and betweengroup analyses, all reported areas of activation were significant using $p<0.05$ FWE corrected at the voxel level with a cluster size greater than 10 voxels. To examine developmental effects that were not due to differences in behavioral performance, we extracted the beta values from the peak voxels of brain regions from the between-group comparison (i.e., the left ventral IFG and left MTG). Then, we partialled out the effect of accuracy in the scanner as a covariate, and ANCOVAs were used to examine the differences in functional activation between groups. All reported results were significant using $p<0.025$ corrected for two brain regions due to our a priori hypothesis.

\section{Effective Connectivity Analysis}

Three regions of interest (ROIs) were chosen from the present study using a more stringent threshold of $p<0.00005$ uncorrected and containing a cluster size greater than or equal to 10 voxels. The threshold was suggested by a previous DCM article to enable the distinction among brain structures that otherwise comprise a single cluster (Bitan et al., 2007; Fan and Chou, 2012). The ROIs were the left ventral IFG (ventral IFG, BA 47), left posterior (MTG, BA 21), and left (FG, BA 37). All ROIs were 6-mm radius spheres centered on the

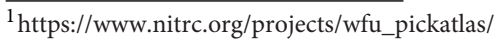

most significant voxel in the individual's activation map within $10-\mathrm{mm}$ from the group maximum.

Effective connectivity analysis was used to examine connections among brain regions, and we used the Dynamic Causal Modelling (DCM) tool in SPM8 (Friston et al., 2003; Penny et al., 2004, 2007; Stephan et al., 2010). Our analysis adopted a two-stage procedure (Bitan et al., 2006; Cao et al., 2008). First, subject-specific DCMs were fully and reciprocally connected, with modulatory (bilinear) effects of the related and the unrelated conditions specified on coupling among all regions. In the DCM models, direct input was specified on FG in the visual modality; intrinsic connections were fully and reciprocally connected between the three ROIs (FG, MTG and IFG). The intrinsic connections between regions showed the interregional influences in the absence of modulating experimental effects (Friston et al., 2003), for example, lack of modulation on meaning judgments in this experiment. We restricted the results of intrinsic connections significant at a level of $p<0.008$ ( $p<0.05$ corrected for six comparisons), tested in a 1-sample $t$-test (Bitan et al., 2005). However, we focused on the modulatory effects, which would be proper to reflect the experimental task (Friston et al., 2003), for example, the interregional influences on meaning judgments. In the second-stage, developmental differences between two groups in the modulatory effects for relatedness conditions were evaluated through three-way analysis of co variance (ANCOVAs): two group (adults, children) by two relatedness condition (related, unrelated) by two coupled connection (top-down, bottom-up) ANCOVAs, with the group as a between-factor, condition/connection as withinfactors, and accuracy as a between-subject covariate. The bottom-up connections from the left FG and the top-down connections from the left IFG/MTG were examined due to previous adult studies using the same semantic task (Fan et al., 2010; Fan and Chou, 2012). This two-stage approach was the same as previous DCM studies of effective connectivity (Bitan et al., 2006; Cao et al., 2008; Kiran et al., 2015; Lahr et al., 2018).

\section{Diffusion MRI Acquisition and Analysis}

DSI was performed using a pulsed-gradient spin-echo diffusion echo-planar imaging sequence with a twice-refocused balanced echo (Reese et al., 2003). The scanning parameters were the following: $\mathrm{TR}=9,600 \mathrm{~ms} ; \mathrm{TE}=130 \mathrm{~ms} ; \operatorname{bmax}=4,000 \mathrm{~s} / \mathrm{mm}^{2}$; slice thickness $=2.5 \mathrm{~mm}$; in-plane spatial resolution $=2.5 \mathrm{~mm}$. To reduce the scan time, 102 diffusion-encoding directions were sampled on the grid points in the half-sphere of the q-space within a radius of 3.6 units (Wedeen et al., 2005). The acquired half-sphere data were projected to fill the other half of the sphere based on the fact that the data in the q-space were real and symmetrical about the origin.

DSI tractography was performed based on a templatebased approach to reconstruct fiber pathways of targeted tracts as described previously (Chen et al., 2015). In brief, Large Deformation Diffeomorphic Metric Mapping (LDDMM) and SPM8 were used to develop a group-specific DSI template from all individuals' DSI datasets (Hsu et al., 2012). Second, 

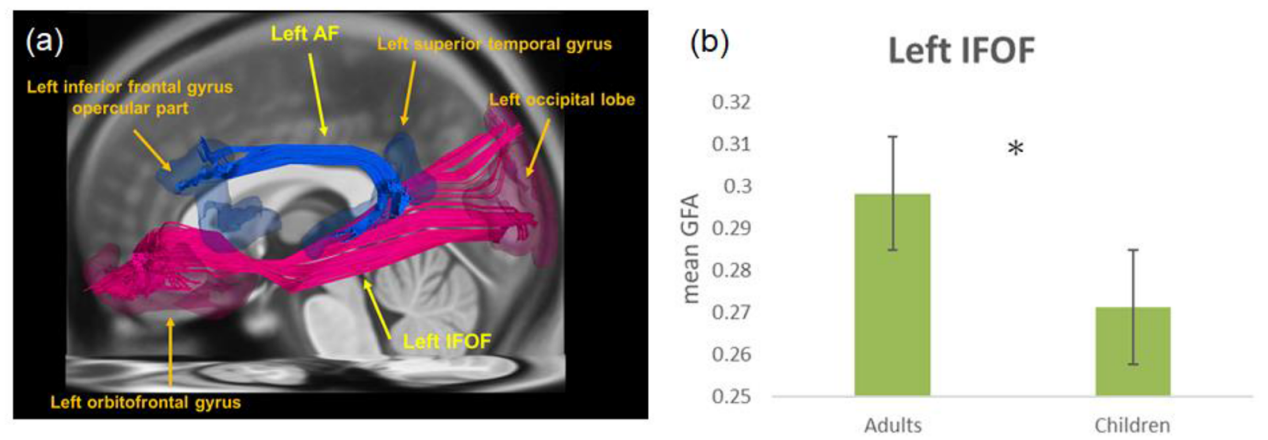

\section{Left IFOF}

FIGURE 1 | (A) The left inferior frontal-occipital fasciculus (IFOF), the arcuate fasciculus (AF), and their corresponding terminal regions in tractography, i.e., the left orbitofrontal gyrus and left occipital lobe for IFOF, as well as the left inferior frontal gyrus (IFG) opercular part and the left superior temporal gyrus (STG) for AF. The left ventral pathway connects the ventral IFG, middle temporal gyrus (MTG), and fusiform gyrus (FG; not shown) via IFOF. (B) The mean generalized fractional anisotropy (GFA) of IFOF in adults was significantly higher than that in children. ${ }^{*} p<0.05$

we normalized the group-specific DSI template to a DSI template in MNI space, which was developed from 122 healthy participants' DSI datasets and coregistered to MNI space (Hsu et al., 2015). The targeted tracts, i.e., the AF and the IFOF, were reconstructed on the DSI template using DSI Studio ${ }^{2}$ (Figure 1A). The reconstructed tracts were transformed from the DSI template in MNI space to an individual's native DSI data through a group-specific DSI template by using LDDMM, and generalized fractional anisotropy (GFA) values were sampled along each of the targeted tracts. Similar to FA, GFA was used to infer the microstructural integrity of white matter fiber tracts (Gorczewski et al., 2009; Fritzsche et al., 2010). In the present study, we selected the GFA values for the AF and IFOF separately and calculated the mean GFA values of each pathway. Also, we treated the effect of accuracy in the scanner as a covariate, and ANCOVAs were used to examine the differences in structural connectivity between groups.

\section{Statistical Analyses}

We used IBM SPSS version 20.0 (SPSS Inc., Chicago, IL, USA) to conduct statistical analysis. The descriptive results were displayed as mean and SD for continuous variables. We conducted a two group (adults, children) by two relatedness condition (related, unrelated) ANOVA to examine the developmental differences between children and adults in behavioral performance. Moreover, ANCOVAs with partialing for accuracy were performed to examine the developmental differences between children and adults in functional activation, structural connectivity, and effective connectivity. Moreover, multiple regressions were used to examine the relationship between age in months and behavioral performance, structural connectivity, and effective connectivity, including accuracy as a between-subject covariate. This approach allowed us to examine age-related increases or decreases in

\footnotetext{
${ }^{2}$ http://dsi-studio.labsolver.org
}

both activation and connectivity that were independent of accuracy differences.

\section{RESULTS}

\section{Behavioral Results}

In the adult group, accuracy (mean \pm SD) for the related and unrelated conditions was $95 \pm 5 \%$ and $99 \pm 2 \%$, respectively. In the child group, accuracy (mean \pm SD) for the related and unrelated conditions was $71 \pm 19 \%$ and $94 \pm 9 \%$, respectively. An ANOVA with two groups (adults, children) by two relatedness condition (related, unrelated), with the group as a betweenfactor and with the condition as within-factors, showed that the main effect of group was significant $\left(F_{(1,50)}=40.30, p<0.01\right)$. The main effect of condition was significant $\left(F_{(1,50)}=45.15\right.$, $p<0.01)$. The interaction of group and condition was significant $\left(F_{(1,50)}=25.51, p<0.01\right)$. To further understand the two-way interaction, $t$-tests showed that adults were more accurate than children in both the related and unrelated conditions $\left(t_{(50)}=6.28\right.$, $p<0.01 ; t_{(50)}=2.64, p<0.01$, respectively).

In the adult group, reaction times (mean $\pm \mathrm{SD}$ ) for the related and unrelated conditions were $824 \pm 154 \mathrm{~ms}$ and $881 \pm 143 \mathrm{~ms}$, respectively. In the child group, reaction times (mean \pm SD) for the related and unrelated conditions were 1,284 $\pm 235 \mathrm{~ms}$ and $1,188 \pm 226 \mathrm{~ms}$, respectively. A two group (adults, children) by two relatedness condition (related, unrelated) ANOVA showed that the main effect of group was significant $\left(F_{(1,50)}=58.08, p<0.01\right)$. The main effect of condition was not significant $\left(F_{(1,50)}=1.05 p=0.31\right)$. The interaction of group and condition was significant $\left(F_{(1,50)}=16.24, p<0.01\right)$. To further understand the two-way interaction, $t$-tests showed that adults were significantly faster than children in the related and unrelated conditions $\left(t_{(50)}=-8.35, p<0.01 ; t_{(50)}=-5.85\right.$, $p<0.01$, respectively).

Accuracy (mean \pm SD) for the baseline conditions in the adults and children was $99 \pm 2 \%$ and $98 \pm 5 \%$, respectively. Reaction times (mean $\pm \mathrm{SD}$ ) for the baseline 


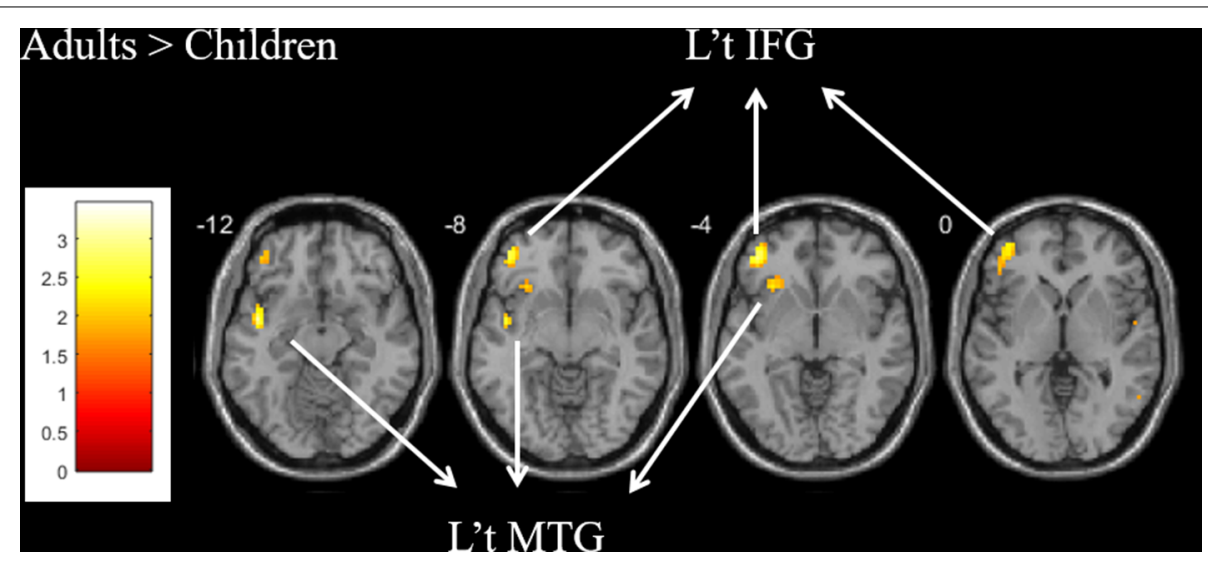

FIGURE 2 | For the contrast of the word vs. baseline condition, greater activation was found in the left ventral IFG and MTG for the adult group as compared with the child group.

conditions in the adults and children were $584 \pm 143 \mathrm{~ms}$ and $786 \pm 141 \mathrm{~ms}$, respectively.

\section{Conventional fMRI Results}

For the contrast of "word vs. baseline condition" within the group, both groups showed common activation in the left ventral IFG (BA 47) and left MTG (BA 21). The direct comparison between groups showed that the adults produced greater activation than children in the left ventral IFG (BA 47; voxels $=54 ; x=-42, y=44, z=-5 ; Z=2.72$ ) and MTG (BA 21 ; voxels $=38 ; x=-57, y=-55, z=-5 ; Z=2.42$; Figure 2 and Supplementary Table S1). The child group showed less activation than the adult group. There was no group difference in the left dorsal IFG. There was no significant correlation between brain activation and age within children.

In order to examine age-related increases or decreases in activation, we extracted beta-values from the left ventral IFG and MTG in the contrast of "word vs. baseline condition," and ANCOVAs were performed with partialing out accuracy as a covariate $(p<0.025$ equal to $p<0.05$ corrected for two comparisons). After partialing out the effect of accuracy, significantly greater beta-values were found in the left ventral IFG $\left(F_{(1,49)}=6.10, p=0.017\right)$ and MTG $\left(F_{(1,49)}=5.75, p=0.020\right)$ for adults relative to children.

\section{Structural Connectivity Differences Between the Adult and Child Groups}

With partialing out the effect of accuracy in the scanner as a covariate, group comparisons revealed a significant difference in the left inferior frontal occipital fasciculus $\left(F_{(1,49)}=23.36\right.$, $p=0.000$ ), showing greater mean GFA for adults as compared to children (Figure 1B). For the left AF, there was no statistically significant group difference $\left(F_{(1,49)}=0.55, p=0.460\right)$.

Within children, there was a positive correlation between mean GFA of the left inferior frontal occipital fasciculus and age $(r=0.574, p=0.003$ ) with partialing out the effect of accuracy in the scanner as a covariate. Conversely, the mean GFA of the left AF was not significantly correlated with age within children $(r=0.061, p=0.774)$.

\section{Effective Connectivity Differences Between the Adult and Child Groups}

Table 1 and Figure 3 presents the posterior means of modulatory effects of the related and unrelated conditions for both the adult and the child groups. One-sample $t$-tests were used to examine whether each modulatory effect was significantly different from zero ( $p<0.008$ equal to $p<0.05$ corrected for six comparisons). Also, all intrinsic connections were significant (Supplementary Table S2).

With accuracy as a covariate, we calculated a two group (adults, children) by two relatedness condition (related, unrelated) by two coupled connection (top-down from the ventral IFG to the FG, bottom-up from the FG to the ventral IFG) ANCOVA to investigate the interaction between the top-down region (ventral IFG) and bottom-up region (FG; Figure 4). The three-way interaction of group (adults, children) $\times$ relatedness condition (related, unrelated) $\times$ coupled connection (top-down from the ventral IFG to the FG, bottom-up from the FG to the ventral IFG) was significant $\left(F_{(1,49)}=5.57, p=0.022\right)$. To further understand the three-way interaction, a two group (adults, children) by two coupled connection (ventral IFG to FG, FG to ventral IFG) ANCOVA was calculated for related and unrelated conditions separately. The analyses revealed that there were significant interactions of group $\times$ direction $\left(F_{(1,49)}=5.99\right.$, $p=0.018)$, and main effect of group $\left(F_{(1,49)}=5.23, p=0.027\right)$ in the related condition, but not in the unrelated condition. To further understand the two-way interaction in the related condition, $t$-tests revealed that the adults showed a significantly larger modulatory effect in the bottom-up connection from the FG to the ventral IFG than in the top-down connection from the ventral IFG to the FG $\left(t_{(26)}=-6.59, p=0.000\right)$ and that the bottom-up effect from the FG to the ventral IFG was stronger in adults as compared to children $\left(t_{(50)}=3.74, p=0.000\right)$. Moreover, with partialing out the effect of accuracy as a covariate, there 
TABLE 1 | The posterior means of the parameter densities on modulatory effects for the adult and child groups for the related and unrelated conditions among all regions (IFG, ventral inferior frontal gyrus; MTG, posterior middle temporal gyrus; FG, fusiform gyrus).

\begin{tabular}{|c|c|c|c|c|c|c|c|}
\hline \multicolumn{4}{|c|}{ Adults related condition } & \multicolumn{4}{|c|}{ Adults unrelated condition } \\
\hline From: & IFG & MTG & $F G$ & From: & IFG & MTG & $F G$ \\
\hline To: & & & & To: & & & \\
\hline IFG & & 0.079 & $1.705^{\star}$ & IFG & & $0.516^{\star}$ & $0.664^{\star}$ \\
\hline MTG & 0.398 & & $1.271^{\star}$ & MTG & 0.047 & & $1.311^{*}$ \\
\hline$F G$ & -0.071 & -0.004 & & $\mathrm{FG}$ & 0.110 & 0.507 & \\
\hline \multicolumn{4}{|c|}{ Children related condition } & \multicolumn{4}{|c|}{ Children unrelated condition } \\
\hline From: & IFG & MTG & $F G$ & From: & IFG & MTG & $F G$ \\
\hline To: & & & & To: & & & \\
\hline IFG & & 0.422 & $0.604^{\star}$ & IFG & & -0.268 & 0.412 \\
\hline MTG & 0.092 & & $0.830^{\star}$ & MTG & 0.195 & & $1.007^{*}$ \\
\hline$F G$ & -0.017 & -0.040 & & $F G$ & -0.028 & 0.276 & \\
\hline
\end{tabular}

Note: ${ }^{*} p<0.008$ ( $p<0.05$ corrected for six comparisons).

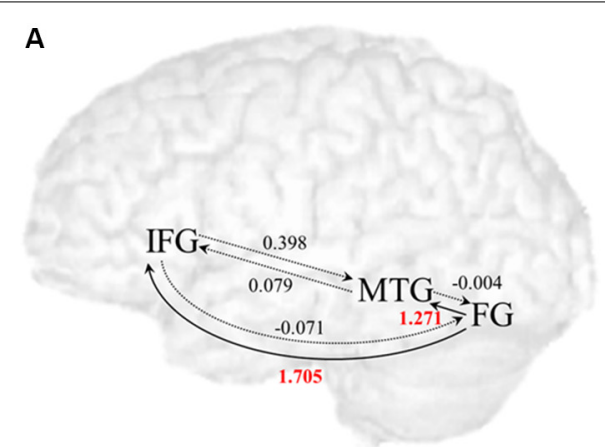

C

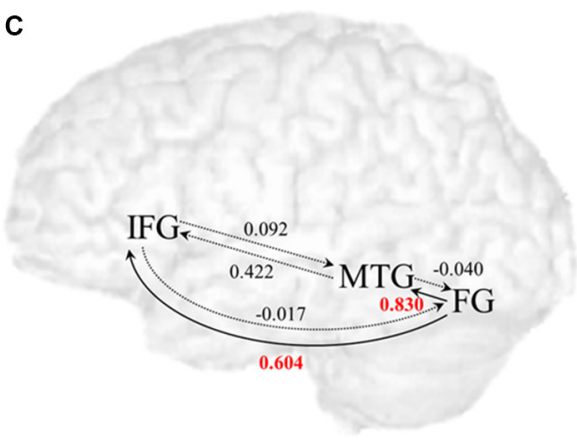

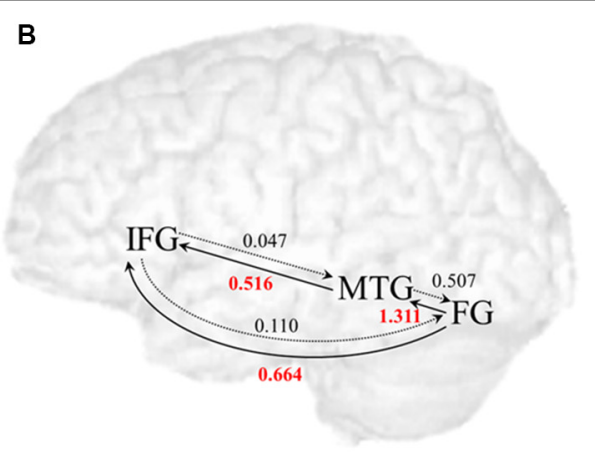

。

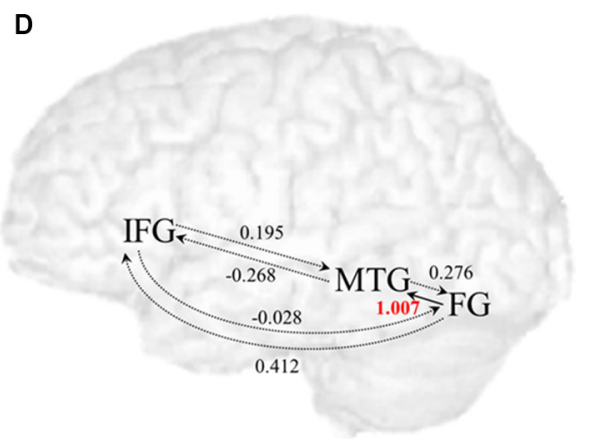

FIGURE 3 | Modulatory effects in the related and unrelated conditions among all regions for the adult and child group. (A) Modulatory effects in the related condition for the adult group. (B) Modulatory effects in the unrelated condition for the adult group. (C) Modulatory effects in the related condition for the child group. (D) Modulatory effects in the unrelated condition for the child group. Solid line arrows indicate significant influences on modulated connections $(p<0.008$ with $p<0.05$ corrected for six comparisons), and dotted line arrows indicate non-significant connections.

was a positive correlation between the modulatory effect from the FG to the ventral IFG and age within children in the related condition $(r=0.431, p=0.031)$.

With accuracy as a covariate, we also calculated a two group (adults, children) by two relatedness condition (related, unrelated) by two coupled connection (top-down from MTG to FG, bottom-up from FG to MTG) ANCOVA to investigate the interaction between the top-down region (MTG) and bottom-up region (FG). The analysis only revealed a marginal main effect of direction $\left(F_{(1,49)}=3.75, p=0.059\right)$ with the bottom-up effect from the FG to MTG being stronger than the top-down effect from the MTG to FG.

\section{Correlations Between Structural Connectivity and Brain Activation/Effective Connectivity}

For the correlation between GFA and activation, partialing out the effect of accuracy as a covariate, there was a positive correlation between mean GFA of the left inferior frontal occipital fasciculus and the ventral IFG $(r=0.337, p=0.016)$. For the correlation between GFA and connection strength, partialing out the effect of accuracy as a covariate, stronger mean GFA of the left inferior frontal occipital fasciculus was positively correlated with the modulatory effect from the FG to the ventral 

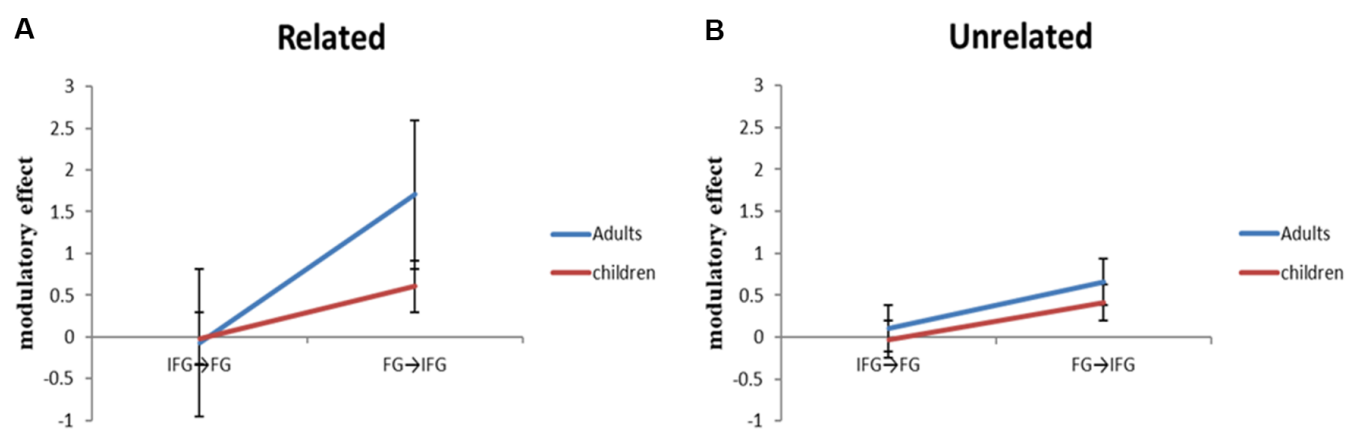

FIGURE 4 | The adult group showed significantly stronger modulatory effects than the child group on the bottom-up connection from the fusiform gyrus (FG) to ventral inferior frontal gyrus (IFG), but not on the top-down connection from the IFG to FG. This difference was found in the related condition, but not in the unrelated condition.

IFG in the related condition $(r=0.313, p=0.025)$, and with the modulatory effect from the FG to the MTG in the related condition $(r=0.297, p=0.036)$.

\section{DISCUSSION}

To the best of our knowledge, the present study is the first to investigate developmental differences in semantic processing regarding brain activation, structural connectivity, and effective connectivity between adults and children. Compared to children, adults showed significantly better behavioral performance, greater functional activation in the left ventral IFG and MTG, and greater mean GFA in the left ventral pathway (IFOF). Moreover, the bottom-up connection from the left FG to the ventral IFG was significantly stronger in adults than that in children for the related condition, but not for the unrelated condition. These findings provide converging evidence to understand the developmental differences between adults and children during semantic processing in Chinese.

In the present study, adults exhibited significantly greater activation in the left ventral IFG (BA 47) and MTG (BA 21) than children, implying maturation in semantic processing via the retrieval or selection of appropriate semantic knowledge (Poldrack et al., 1999; Wagner et al., 2001). Previous studies have suggested that the ventral IFG is associated with access, retrieval, selection, and gating of semantic information, while the MTG may play a role in storing conceptual features that are associated with lexical representations (Hickok and Poeppel, 2007; Martin, 2007; Sonty et al., 2007). In the present study, however, adults did not show increased activation in the dorsal IFG as compared to children. This finding implies that the dorsal IFG may not play a significant role in the developmental differences of semantic processing.

In the present study, a greater mean GFA of the left ventral pathway (IFOF) was found in adults; however, we did not find a group difference in the left dorsal pathway (AF). The findings suggest maturation in the ventral pathway. Previous studies have found that the ventral pathway is involved in lexical-semantic processing (Glasser and Rilling, 2008; Saur et al., 2008), and the dorsal pathway (AF) is involved in phonological processing (Saur et al., 2008). For language functions, both pathways are not mutually exclusive but rather work in parallel (Rauschecker and Scott, 2009). Also, previous DTI studies have proposed that age-related increases in FA may be involved in axonal myelination for both the dorsal and ventral pathways (Perani et al., 2011). However, we only found a positive correlation between the mean GFA of the left ventral pathway (IFOF) and age within children, suggesting an age-related increase in microstructural integrity of the ventral pathways. As their age increased, elder children may have greater microstructural integrity in the ventral pathways as compared to younger children.

From effective connectivity results, we found that the bottom-up connection from the left FG to ventral IFG was significantly stronger in adults than that in children for the related condition, but not for the unrelated condition. Previous studies have suggested that the ventral IFG is associated with retrieval or selection of appropriate semantic knowledge (Thompson-Schill et al., 1999; Wagner et al., 2001), while the FG is involved in visual-orthographic processing (Nakamura et al., 2000; Lin et al., 2007). Orthographic processing is generally attributed to the FG, which has been reliably shown to be responsible for visually presented words (McCandliss et al., 2003). Moreover, older children may more rely on orthographic pattern separation showing greater activation in the FG than younger children (McNorgan et al., 2011). Taken together, the left ventral IFG is implicated in controlling semantic retrieval, while the FG is implicated in processing orthographic representations (Lau et al., 2008). Therefore, our finding of a stronger FG-ventral IFG connection in adults suggests that orthographic processing in the FG may have a strong link with semantic retrieval in the ventral IFG in adults than in children. More importantly, we found a positive correlation between the FG-ventral IFG connection and age within children. This correlation provides further evidence of the mapping from orthography to semantics with age during semantic processing in Chinese.

The findings and interpretations of the present study should be considered in light of its limitation. Although there were no 
significant differences of head motion in translation and rotation between children and adults (x-translation: $t_{(50)}=0.63, p>0.05$; y-translation: $t_{(50)}=-1.18, p>0.05$; z-translation: $t_{(50)}=-1.15$, $p>0.05$; pitch: $t_{(50)}=1.37, p>0.05$; roll: $t_{(50)}=1.94, p>0.05$; yaw: $t_{(50)}=-0.03, p>0.05$, respectively), the age-related head size might be a potential confound. Therefore, a larger sample with a wider age range from childhood to adulthood is needed to further evaluate the structure and function of the neural mechanism underlying semantic processing across different developmental stages. Moreover, in addition to the crosssectional studies, a prospective longitudinal study investigating the trajectory of developmental changes in semantic processing is warranted.

In conclusion, combining fMRI, DSI, and DCM provides a complete insight into the developmental differences between adults and children during semantic processing in Chinese. Our findings suggest that age-related maturation in brain activation (ventral IFG and MTG), structural connectivity (IFOF), and effective connectivity (from FG to ventral IFG) might be associated with the bottom-up influences of orthographic representations on retrieving semantic representations for processing Chinese characters. Our results clearly contribute to our knowledge about developmental differences, which are not only associated with structural maturation but also with functional brain activation. Most importantly, our findings of effective connectivity revealed that adults might be better able to utilize visual-orthographic word information of the fusiform cortex during Chinese meaning judgments. These findings might advance our knowledge of the communication between brain regions for language functions, especially for adults and children in Chinese.

\section{DATA AVAILABILITY STATEMENT}

The datasets generated for the present study are available on request to the corresponding author.

\section{REFERENCES}

Bitan, T., Booth, J. R., Choy, J., Burman, D. D., Gitelman, D. R., and Mesulam, M.M. (2005). Shifts of effective connectivity within a language network during rhyming and spelling. J. Neurosci. 25, 5397-5403. doi: 10.1523/JNEUROSCI. 0864-05.2005

Bitan, T., Burman, D. D., Lu, D., Cone, N. E., Gitelman, D. R., Mesulam, M.-M., et al. (2006). Weaker top-down modulation from the left inferior frontal gyrus in children. NeuroImage 33, 991-998. doi: 10.1016/j.neuroimage.2006.07.007

Bitan, T., Cheon, J., Lu, D., Burman, D. D., and Booth, J. R. (2009). Developmental increase in top-down and bottom-up processing in a phonological task: an effective connectivity, fMRI study. J. Cogn. Neurosci. 21, 1135-1145. doi: 10.1162/jocn.2009.21065

Bitan, T., Cheon, J., Lu, D., Burman, D. D., Gitelman, D. R., Mesulam, M.-M., et al. (2007). Developmental changes in activation and effective connectivity in phonological processing. NeuroImage 38, 564-575. doi: 10.1016/j.neuroimage. 2007.07.048

Booth, J. R., Lu, D., Burman, D. D., Chou, T.-L., Jin, Z., Peng, D.-L., et al. (2006). Specialization of phonological and semantic processing in Chinese word reading. Brain Res. 1071, 197-207. doi: 10.1016/j.brainres.2005. 11.097

\section{ETHICS STATEMENT}

The studies involving human participants were reviewed and approved by National Taiwan University Hospital Research Ethics Committee. Written informed consent to participate in this study was provided by the participants' legal guardian/next of kin.

\section{AUTHOR CONTRIBUTIONS}

L-YF, W-YT, and T-LC designed research. L-YF, Y-CL, Y-CH, and Y-JC decided upon and performed the analyses. L-YF drafted the main manuscript text. All authors reviewed the manuscript and approved it for submission.

\section{FUNDING}

This work was supported by National Science Council. (NSC 101-2627-B-002-001) in part by the Department of Medical Imaging and 3T MRI Lab at National Taiwan University Hospital.

\section{ACKNOWLEDGMENTS}

We thank the participants of the Annual Meeting for Society for the Neurobiology of Language (SNL) conference 2013 "Developmental changes of structural connectivity and effective connectivity in semantic judgments of Chinese characters" for their very valuable comments and questions on the poster version of this manuscript.

\section{SUPPLEMENTARY MATERIAL}

The Supplementary Material for this article can be found online at: https://www.frontiersin.org/articles/10.3389/fnhum.2020.002 33/full\#supplementary-material.

Brauer, J., Anwander, A., Perani, D., and Friederici, A. D. (2013). Dorsal and ventral pathways in language development. Brain Lang. 127, 289-295. doi: 10.1016/j.bandl.2013.03.001

Burock, M. A., Buckner, R. L., Woldorff, M. G., Rosen, B. R., and Dale, A. M. (1998). Randomized event-related experimental designs allow for extremely rapid presentation rates using functional MRI. Neuroreport 9, 3735-3739. doi: 10.1097/00001756-199811160-00030

Cao, F., Bitan, T., and Booth, J. R. (2008). Effective brain connectivity in children with reading difficulties during phonological processing. Brain Lang. 107, 91-101. doi: 10.1016/j.bandl.2007.12.009

Cao, F., Khalid, K., Lee, R., Brennan, C., Yang, Y., Li, K., et al. (2011). Development of brain networks involved in spoken word processing of mandarin chinese. NeuroImage 57, 750-759. doi: 10.1016/j.neuroimage.2010.09.047

Chen, Y. J., Lo, Y. C., Hsu, Y. C., Fan, C. C., Hwang, T. J., Liu, C. M., et al. (2015). Automatic whole brain tract-based analysis using predefined tracts in a diffusion spectrum imaging template and an accurate registration strategy. Hum. Brain Mapp. 36, 3441-3458. doi: 10.1002/hbm.22854

Chou, T.-L., Booth, J. R., Bitan, T., Burman, D. D., Bigio, J. D., Cone, N. E., et al. (2006). Developmental and skill effects on the neural correlates of semantic processing to visually presented words. Hum. Brain Mapp. 27, 915-924. doi: $10.1002 / \mathrm{hbm} .20231$ 
Chou, T.-L., Chen, C.-W., Fan, L.-Y., Chen, S.-Y., and Booth, J. R. (2009a). Testing for a cultural infl uence on reading for meaning in the developing brain: the neural basis of semantic processing in Chinese children. Front. Hum. Neurosci. 3:27. doi: 10.3389/neuro.09.027.2009

Chou, T.-L., Chen, C.-W., Wu, M.-Y., and Booth, J. R. (2009b). The role of inferior frontal gyrus and inferior parietal lobule in semantic processing of Chinese characters. Exp. Brain Res. 198, 465-475. doi: 10.1007/s00221-009-1942-y

Cohen, L., Jobert, A., Bihan, D. L., and Dehaene, S. (2004). Distinct unimodal and multimodal regions for word processing in the left temporal cortex. NeuroImage 23, 1256-1270. doi: 10.1016/j.neuroimage.2004.07.052

Devereux, B. J., Clarke, A., Marouchos, A., and Tyler, L. K. (2013). Representational similarity analysis reveals commonalities and differences in the semantic processing of words and objects. J. Neurosci. 33, 18906-18916. doi: 10.1523/JNEUROSCI.3809-13.2013

Dong, Y., Nakamura, K., Okada, T., Hanakawa, T., Fukuyama, H., Mazziotta, J. C., et al. (2005). Neural mechanisms underlying the processing of Chinese words: an fMRI study. Neurosci. Res. 52, 139-145. doi: 10.1016/j.neures.2005.02.005

Fan, L.-Y., and Chou, T.-L. (2012). Hierarchical model comparisons on effective connectivity in semantic judgments of chinese characters. Chinese J. Psychol. 54, 1-16. doi: 10.6129/CJP.2012.5401.03

Fan, L.-Y., Lee, S.-H., and Chou, T.-L. (2010). Interaction between brain regions during semantic processing in Chinese adults. Lang. Linguist. 11, 159-182.

Friston, K. J., Harrison, L., and Penny, W. (2003). Dynamic causal modelling. NeuroImage 19, 1273-1302. doi: 10.1016/s1053-8119(03)00202-7

Fritzsche, K. H., Laun, F. B., Meinzer, H. P., and Stieltjes, B. (2010). Opportunities and pitfalls in the quantification of fiber integrity: what can we gain from Q-ball imaging? NeuroImage 51, 242-251. doi: 10.1016/j.neuroimage.2010.02.007

Glasser, M. F., and Rilling, J. K. (2008). DTI tractography of the human Brain's language pathways. Cereb. Cortex 18, 2471-2482. doi: 10.1093/cercor/bhn011

Gorczewski, K., Mang, S., and Klose, U. (2009). Reproducibility and consistency of evaluation techniques for HARDI data. MAGMA 22, 63-70. doi: 10.1007/s10334-008-0144-0

Hickok, G., and Poeppel, D. (2007). The cortical organization of speech processing. Nat. Rev. Neurosci. 8, 393-402. doi: 10.1038/nrn2113

Hsu, Y. C., Hsu, C. H., and Tseng, W. Y. I. (2012). A large deformation diffeomorphic metric mapping solution for diffusion spectrum imaging datasets. NeuroImage 63, 818-834. doi: 10.1016/j.neuroimage.2012.07.033

Hsu, Y. C., Lo, Y. C., Chen, Y. J., Wedeen, V. J., and Isaac Tseng, W. Y. (2015). NTU-DSI-122: a diffusion spectrum imaging template with high anatomical matching to the ICBM-152 space. Hum. Brain Mapp. 36, 3528-3541. doi: $10.1002 / \mathrm{hbm} .22860$

Hue, C.-W., Kao, C.-H., and Lo, M. (2005). Association Norms for 600 Chinese Characters. Taiwanese Psychological Association. Taipei.

Josephs, O., and Henson, R. N. A. (1999). Event-related functional magnetic resonance imaging: modelling, inference and optimization. Philos. Trans. R Soc. Biol. Sci. 354, 1215-1228. doi: 10.1098/rstb.1999.0475

Kiran, S., Meier, E. L., Kapse, K. J., and Glynn, P. A. (2015). Changes in task-based effective connectivity in language networks following rehabilitation in post-stroke patients with aphasia. Front. Hum. Neurosci. 9:316. doi: 10.3389/fnhum.2015.00316

Lahr, J., Minkova, L., Tabrizi, S. J., Stout, J. C., Klöppel, S., Scheller, E., et al. (2018). Working memory-related effective connectivity in Huntington's disease patients. Front. Neurol. 4:370. doi: 10.3389/fneur.2018.00370

Lau, E. F., Phillips, C., and Poeppel, D. (2008). A cortical network for semantics: (De)constructing the N400. Nat. Rev. Neurosci. 9, 920-933. doi: $10.1038 / \mathrm{nrn} 2532$

Lebel, C., Walker, L., Leemans, A., Phillips, L., and Beaulieua, C. (2008). Microstructural maturation of the human brain from childhood to adulthood. NeuroImage 40, 1044-1055. doi: 10.1016/j.neuroimage.2007.12.053

Lee, S.-H., Chen, S.-Y., and Chou, T.-L. (2009). Effects of vocabulary sizes on semantic processing to Chinese characters between fifth graders and adults. Formos. J. Ment. Health 22, 345-382. doi: 10.30074/FJMH.200912_22(4).0001

Lin, C. Y., Xiao, Z. W., Shen, L., Zhang, J. X., and Weng, X. C. (2007). Similar brain activation patterns for writing logographic and phonetic symbols in Chinese. Neuroreport 18, 1621-1625. doi: 10.1097/wnr.0b013e3282f0405b

Liu, L., Deng, X., Peng, D., Cao, F., Ding, G., Jin, Z., et al. (2009). Modalityand task-specific brain regions involved in chinese lexical processing. J. Cogn. Neurosci. 21, 1473-1487. doi: 10.1162/jocn.2009.21141
Liu, L., Vira, A., Friedman, E., Minas, J., Bolger, D., Bitan, T., et al. (2010). Children with reading disability show brain differences in effective connectivity for visual, but not auditory word comprehension. PLoS One 5:e13492.doi: 10.1371/journal.pone.0013492

Martin, A. (2007). The representation of object concepts in the brain. Annu. Rev. Psychol. 58, 25-45. doi: 10.1146/annurev.psych.57.102904. 190143

McCandliss, B. D., Cohen, L., and Dehaene, S. (2003). The visual word form area: expertise for reading in the fusiform gyrus. Trends Cogn. Sci. 7, 293-299. doi: 10.1016/s1364-6613(03)00134-7

McNorgan, C., Alvarez, A., Bhullar, A., Gayda, J., and Booth, J. R. (2011). Prediction of reading skill several years later depends on age and brain region: implications for developmental models of reading. J. Neurosci. 31, 9641-9648. doi: 10.1523/JNEUROSCI.0334-11.2011

Mei, L., Xue, G., Lu, Z. L., He, Q., Zhang, M., Xue, F., et al. (2013). Orthographic transparency modulates the functional asymmetry in the fusiform cortex: an artificial language training study. Brain Lang. 125, 165-172. doi: 10.1016/j. bandl.2012.01.006

Nakamura, K., Honda, M., Okada, T., Hanakawa, T., Toma, K., Fukuyama, H., et al. (2000). Participation of the left posterior inferior temporal cortex in writing and mental recall of kanji orthography-a functional MRI study. Brain 123, 954-967. doi: 10.1093/brain/123.5.954

Olesen, P. J., Nagy, Z., Westerberg, H., and Klingberg, T. (2003). Combined analysis of DTI and fMRI data reveals a joint maturation of white and grey matter in a fronto-parietal network. Cogn. Brain Res. 18, 48-57. doi: 10.1016/j. cogbrainres.2003.09.003

Paus, T., Keshavan, M., and Giedd, J. N. (2008). Why do many psychiatric disorders emerge during adolescence? Nat. Rev. Neurosci. 9, 947-957. doi: $10.1038 / \mathrm{nrn} 2513$

Penny, W. D., Friston, K. J., Ashburner, J., Kiebel, S., and Nichols, T. (2007). Statistical Parametric Mapping: The Analysis of Functional Brain Images. Elsevier: Academic Press.

Penny, W. D., Stephan, K. E., Mechelli, A., and Friston, K. J. (2004). Comparing dynamic causal models. NeuroImage 22, 1157-1172. doi: 10.1016/j.neuroimage. 2004.03.026

Perani, D., Saccuman, M. C., Scifo, P., Awander, A., Spada, D., Baldoli, C., et al. (2011). Neural language networks at birth. Proc. Natl. Acad. Sci. U S A 108 , 16056-16061. doi: 10.1073/pnas.1102991108

Poldrack, R. A., Wagner, A. D., Prull, M. W., Desmond, J. E., Glover, G. H., and Gabrieli, J. D. (1999). Functional specialization for semantic and phonological processing in the left inferior prefrontal cortex. NeuroImage 10, 15-35. doi: 10.1006/nimg.1999.0441

Rauschecker, J. P., and Scott, S. K. (2009). Maps and streams in the auditory cortex: nonhuman primates illuminate human speech processing. Nat. Neurosci. 12, 718-724. doi: 10.1038/nn.2331

Reese, T. G., Heid, O., Weisskoff, R. M., and Wedeen, V. J. (2003). Reduction of eddy-current-induced distortion in diffusion MRI using a twice-refocused spin echo. Magn. Reson. Med. 49, 177-182. doi: 10.1002/mrm.10308

Rossell, S. L., Bullmore, E. T., Williams, S. C. R., and David, A. S. (2001). Brain activation during automatic and controlled processing of semantic relations: a priming experiment using lexical decision. Neuropsychologia 39, 1167-1176. doi: 10.1016/s0028-3932(01)00049-5

Saggar, M., Shelly, E. W., Lepage, J. F., Hoeft, F., and Reiss, A. L. (2014). Revealing the neural networks associated with processing of natural social interaction and the related effects of actor-orientation and face-visibility. NeuroImage 84, 648-656. doi: 10.1016/j.neuroimage.2013.09.046

Saur, D., Kreher, B. R. W., Schnell, S., Kümmerer, D., Kellmeyer, P., Vry, M.-S., et al. (2008). Ventral and dorsal pathways for language. Proc. Natl. Acad. Sci. U S A 18, 18035-18040. doi: 10.1073/pnas.0805234105

Schmithorst, V. J., and Yuan, W. H. (2010). White matter development during adolescence as shown by diffusion MRI. Brain Cogn. 72, 16-25. doi: 10.1016/j. bandc.2009.06.005

Sinica, C. (1998). Academia Sinica Balanced Corpus (Version 3) Taipei. Taiwan: Academia Sinica.

Sonty, S. P., Mesulam, M. M., Weintraub, S., Johnson, N. A., Parrish, T. B., and Gitelman, D. R. (2007). Altered effective connectivity within the language network in primary progressive aphasia. J. Neurosci. 27, 1334-1345. doi: 10.1523/JNEUROSCI.4127-06.2007 
Stephan, K. E., Penny, W. D., Moran, R. J., Ouden, H. E. M. D., Daunizeau, J., and Friston, K. J. (2010). Ten simple rules for dynamic causal modeling. NeuroImage 49, 3099-3109. doi: 10.1016/j.neuroimage.2009.11.015

Thompson-Schill, S. L., D’Esposito, M., and Kan, I. P. (1999). Effects of repetition and competition on activity in left prefrontal cortex during word generation. Neuron 23, 513-522. doi: 10.1016/s0896-6273(00)80804-1

Wagner, A. D., Paré-Blagoev, E. J., Clark, J., and Poldrack, R. A. (2001). Recovering meaning: left prefrontal cortex guides controlled semantic retrieval. Neuron 31, 329-338. doi: 10.1016/s0896-6273(01)00359-2

Walter, E., and Dassonville, P. (2011). Activation in a frontoparietal cortical network underlies individual differences in the performance of an embedded figures task. PLoS One 6:e20742. doi: 10.1371/journal.pone. 0020742

Wedeen, V. J., Hagmann, P., Tseng, W.-Y. I., Reese, T. G., and Weisskoff, R. M. (2005). Mapping complex tissue architecture with diffusion spectrum magnetic resonance imaging. Magn. Reson. Med. 54, 1377-1386. doi: 10.1002/mrm. 20642

Werring, D. J., Clark, C. A., Barker, G. J., Miller, D. H., Parker, G. J. M., Brammer, M. J., et al. (1998). The structural and functional mechanisms of motor recovery: complementary use of diffusion tensor and functional magnetic resonance imaging in a traumatic injury of the internal capsule. J. Neurol. Neurosurg. Psychiatry 65, 863-869. doi: 10.1136/jnnp. 65.6.863

Werring, D. J., Clark, C. A., Parker, G. J. M., Miller, D. H., Thompson, A. I., and Barker, G. J. (1999). A direct demonstration of both structure and function in the visual system: combining diffusion tensor imaging with functional magnetic resonance imaging. NeuroImage 9, 352-361. doi: 10.1006/nimg. 1999.0421

Wieshmann, U. C., Krakow, K., Symms, M. R., Parker, G. J. M., Clark, C. A., Barker, G. J., et al. (2001). Combined functional magnetic resonance imaging and diffusion tensor imaging demonstrate widespread modified organisation in malformation of cortical development. J. Neurol. Neurosurg. Psychiatry 70, 521-523. doi: 10.1136/jnnp.70.4.521

Wu, J.-T., and Chen, H.-C. (2000). Evaluating semantic priming and homophonic priming in recognition and naming of Chinese characters. Chinese J. Psychol. 42, 65-86. doi: 10.6129/CJP.2003.4501.05

Wu, J.-T., and Liu, I.-M. (1987). Exploring the Phonetic and Semantic Features of Chinese words. Taipei: Taiwan National Science Council. Technical Report NSC75-0301-H002-024.

Conflict of Interest: The authors report no biomedical financial interests or potential conflicts of interest.

The handling Editor declared a past co-authorship with one of the authors W-YT.

Copyright (C) $2020 \mathrm{Fan}, \mathrm{Lo}, \mathrm{Hsu}$, Chen, Tseng and Chou. This is an open-access article distributed under the terms of the Creative Commons Attribution License (CC BY). The use, distribution or reproduction in other forums is permitted, provided the original author(s) and the copyright owner(s) are credited and that the original publication in this journal is cited, in accordance with accepted academic practice. No use, distribution or reproduction is permitted which does not comply with these terms. 